\title{
CAPACITY-ORIENTED PRODUCTION PLANNING IN CASE OF A SINGLE BOTTLE-NECK
}

\author{
Roland Bemelmans
}

Eindhoven University of Technologv, Den Dolech 2, Postbox 513, 5600 MB Eindhoven (The Netherlands)

\section{INTRODUCTION}

The Material Requirements Planning (MRP) approach of production and inventory control is basically product-oriented: the MRP controlled system only responds to demand fluctuations and not to capacity fluctuations. The capacity aspect has to be taken care of in the Master Production Schedule (MPS), but the MPS part of most MRP systems is rather underdeveloped.

In this paper we develop a more capacityoriented system of production and inventory control and we compare its performance with the performance of a product-oriented system. The simplest situation that can be used to compare the capacity-oriented approach with the product-oriented approach is the situation in which we only have one bottlèneck. Such a situation can be modelled as a single-machine, multi-product planning problem.

Some research has been done on this problem already (see e.g. Bemelmans and Wijngaard $[2,3])$. The results from that research show that the capacity-oriented approach clearly outperforms the product-oriented approach in several cases. A simple criterion is derived for when to use each of the approaches.

However, the research done so far needs extension. It is not only assumed that there is just one single bottleneck, but also it is as- sumed that there are only few products and that the average demand in the long run is the same for all products. Thus there are only short term differences (different demand predictions and realizations) between the products (see Bemelmans [4]). In this paper we consider the case with many non-identical products. In section 2 we describe the model that we use. It is not possible to determine an optimal strategy for this case. In section 3 we develop a heuristic approach, taking into account the just mentioned results. The approach is based on the decomposition of the complete set of products in fastmovers and slowmovers. For the slowmovers, a productoriented approach is used (see section 3.1). The capacity aspect is included in the control of fastmovers (section 3.2). In section 3.3 we give a criterion of where to split the set of products.

\section{DESCRIPTION OF THE MODEL}

Suppose we have $N$ products that make use of the same limited resource (see Fig. 1).

The demand for product $j$ follows a Poisson process with parameter $\lambda_{j}$. Let $\lambda=\Sigma_{j=1}^{N} \lambda_{j}$. The distribution of the total average demand over the products is rather unimportant, but to make things more specific we assume $\lambda_{j}=\lambda / j$. There is no correlation between the products.

Let $I_{j}(t)$ denote the inventory for product 
$j$ at time $t$. The inventory may be negative, since we allow for backordering. The cost that is related to these inventories is: linear inventory holding cost (rate $a$ ) and linear backorder cost (rate $b$ ) as in (1).

$\Sigma_{j=1}^{N}\left[a I_{j}^{+}(t)+b I_{j}^{+}(t)\right]$

where $I^{+}=\max (0, I)$ and $I^{-}=\max (0,-I)$.

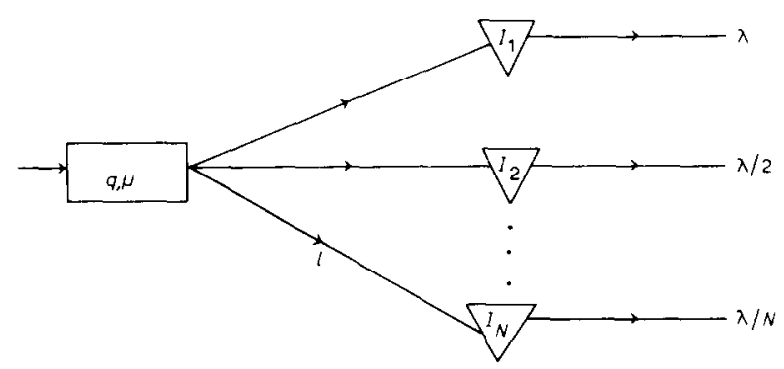

Fig. 1. The single-machine, multi-product planning problem.

It is our aim to minimize the (expected) cost per unit of time. In order to reach this goal, we may decide to start a production run for one of the products at certain production instants. These production instants follow a Poission process with parameter $\mu$. If a production run is started then always a batch of size $q$ is produced. (Thus we assume that the lot-size determination is decomposed from the production planning problem).

The batch that is produced will arrive at the stock-point of the product, for which the run has been started, after $l$ units of time. This $l$ can be seen as a lead time (or transportation time). Notice that this means that there may sometimes be several production runs in process. This can be seen in Fig. 2.

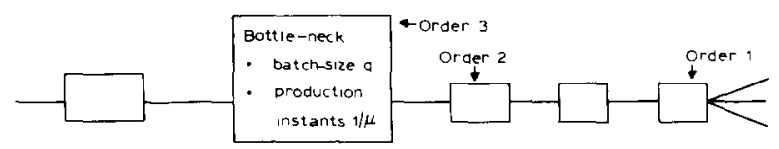

Fig. 2. Production process that is characterized by a single bottleneck.

\section{HEURISTIC APPROACH}

It is not possible to determine an optimal strategy, because of numerical problems. Therefore we introduce a heuristic approach. Non-numerical reasons to use a heuristic approach are:

- usually one encounters systems in practice that do not fit in an exact, existing theoretic model. Yet one approximates the system by such a model. Therefore it is not sensible to put a lot of effort in trying to find an optimal strategy in the theoretical model since in reality it will not be optimal.

- the optimal strategy will not be very transparant. This will certainly lead to implementation problems.

The heuristic developed here is based on the results for the case with few identical products. These results indicate that

- product-oriented strategies are not good in situations with a tight capacity

- the performance of capacity-oriented strategies decreases with an increasing number of products.

Therefore we decompose the complete set of products in fastmovers and slowmovers. There have to be relatively few fastmovers (products 1 to $N_{a}$ ) and the total capacity usage of these fastmovers must be high. Then there are relatively many slowmovers (products $N_{a}+1$ to $N$ ), each having only a minor claim on the capacity.

With this decomposition between slowmovers and fastmovers, we are able to develop a feasible capacity-oriented approach. We will get strategies that not only respond to fluctuations in the demand for individual products, but also to capacity fluctuations. So we will consider the stored capacity aspect of the inventory if making a production decision. Notice that the inventory for a certain product is only effective as stored capacity if there is a production for that product (otherwise) before the inventory for one of the other products triggers a production. By its defini- 
tion a demand for a fastmover (and therefore a production) occurs much more frequently than for a slowmover. Therefore, if we store some capacity in the form of inventory then we should do so for fastmovers and not for slowmovers.

The heuristic that we propose therefore is to start a production run for a slowmover if the situation with respect to the individual product suggests so, while a production run for a slowmover is started if the capacity situation of the system (that is measured by the aggregate inventory of the fastmovers) suggests so. In the next subsections we will describe the heuristic for both slowmovers and fastmovers in more detail.

The approach implies that the slowmovers have priority in order to avoid that they suffer much from the capacity restriction, while this capacity restriction is not taken into account in the control of slowmovers.

\subsection{Slowmovers}

The production instants for slowmovers follow a Poisson process with parameter $\mu$. Since the total capacity usage by slowmovers is only small, we can decompose the different slowmovers by neglecting the capacity restriction. The production strategy for product $j$ will have the following form (if we let $I p o s_{j}$ denote the inventory position for product $j$ ):

"produce product $j$ iff $I p o s_{j} \leqslant I_{j}^{(0)}$,"

where $I_{j}^{(0)}$ is a predetermined level.

If it would happen that there is more than one slowmover that triggers a production run, then we have to make a choice among them. A reasonable choice proves to be (see e.g. Bemelmans [4]) to assign the production run to the products in such a way that the first cost that is influenced by the choice will be minimized (for a further reference on stochastic allocation rules the reader is referred to Agnihothri e.a. [1]). Thus we minimize expression (2) over $j$.

$$
\begin{aligned}
& \Sigma_{k=0}^{\infty}\left\{\left[a\left(l p o s_{j}+q-k\right)^{+}+b\left(I p o s_{j}+q-k\right)^{-}\right]\right. \\
& \left.-\left[a\left(I p o s_{j}-k\right)^{+}+b\left(I p o s_{j}-k\right)^{-}\right]\right\} \\
& \text {- } \frac{(l \lambda / j)^{k}}{k !} \mathrm{e}^{-l \lambda / j}
\end{aligned}
$$

We will now describe a way to determine $I_{j}^{(0)}$ under the assumption that the slowmovers do not interfere. Let $T_{j}^{(0)}\left(\right.$ Ipos $\left._{j}\right)$ and $C_{f_{j}}^{(0)}$ $\left({ }^{\prime} \cos _{j}\right)$ be respectively the expected time and cost, until the inventory position of product equals $I_{j}^{(0)}$ if it equals $I$ Ipos $S_{j}$ now. If we suppress the index $I_{j}^{(0)}$ then it will be clear that $T\left(I p o s_{j}\right)$ and $C\left(\operatorname{Ipos}_{j}\right)$ satisfy the following recurrence relations.

$$
\left[\begin{array}{rl}
T\left(I p o s_{j}\right)= & \frac{1}{\frac{\lambda}{j}+\mu} \cdot\left(1+\frac{\lambda}{j} T\left(\text { Ipos }_{j}-1\right)+\mu T\left(I \operatorname{lpos}_{j}\right)\right] \\
& \forall \text { Ipos }_{j}>I_{j}^{(0)} \\
T\left(I_{j}^{(0)}\right)= & 0 \\
T\left(\text { Ipos }_{j}\right)= & \frac{1}{\frac{\lambda}{j}+\mu} \cdot\left[1+\frac{\lambda}{j} T\left(\text { Ipos }_{j}-1\right)+\mu T\left(\text { Ipos }_{j}+q\right)\right] \\
& \forall \text { Ipos }_{j}<I_{j}^{(0)}
\end{array}\right.
$$

and

$$
\left\{\begin{aligned}
C\left(I p o s_{j}\right)= & \frac{1}{\lambda} \cdot\left\{\Sigma_{k=0}^{\infty}\left[a\left(I p o s_{j}-k\right)^{+}+b\left(I p o s_{j}-k\right)^{-}\right]\right. \\
& \left.\frac{(I \lambda / j)^{k}}{k !} \mathrm{e}^{-l \lambda / j}+\frac{\lambda}{j} C\left(I p o s_{j}-1\right)+\mu C(I p o s)\right\} \\
& \left.\forall I I_{j}^{(0)}\right)= \\
C\left(I p o s_{j}>I_{j}\right)= & \frac{1}{\lambda} \cdot\left\{\Sigma_{k=0}^{\infty}\left[a\left(I p o s_{j}-k\right)^{+}+b\left(I p o s_{j}-k\right)^{-}\right]\right. \\
& \frac{\lambda}{j}+\mu \\
& \left.\cdot \frac{(I \lambda / j)^{k}}{k !} \mathrm{e}^{-\lambda \lambda / j}+\frac{\lambda}{j} C\left(I p o s_{j}-1\right)+\mu C(I p o s+q)\right\} \\
& \forall I p o s_{j}<I_{j}^{(0)}
\end{aligned}\right.
$$

These one-dimensional recurrence relations 
can be solved relatively easy. Using the ergodic theorem for averages (see e.g. Cohen [5]), we then also find the average cost per period (say $g$ ) for a given choice of $I_{j}^{(0)}$ as follows:

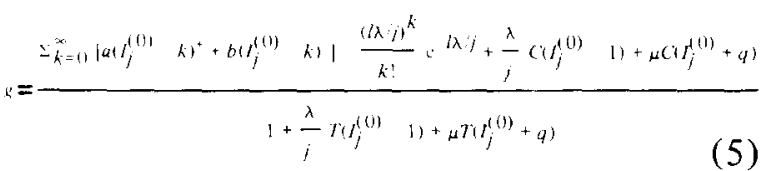

Notice that the denominator of (5) does not depend on the choice of $r_{j}^{(0)}$. Therefore we only have to solve (4) for several choices of $I_{j}^{(0)}$ if we want to find an optimal choice of $I_{j}^{(0)}$.

\subsection{Fastmovers}

For the control of fastmovers, the capacity. aspect of the system is considered. Thar means that the decision whether or not to produce has to depend on the total inventory in the sense of stored capacity. Since the time until the next production instant does not depend on which fastmover one produces, the sum of the individual inventory positions is the best measure for the amount of stored capacity. So we have to define the aggregate inventory position as Ipos: $=\sum_{j=1}^{N a} I_{p o s}$, and we have to consider production strategies for fastmovers of the following form:

"produce a fastmover iff $I p o s \leqslant \Gamma^{(0), "}$

for certain, predetermined level $I^{(0)}$.

If a production run is started then (by the same argument as for slowmovers) this run will be assigned to a fastmover on ground of (2).

An optimal choice of $I^{(0)}$ depends on

(1) the distribution of the aggregate inventory position over the individual fastmovers

(2) the demand process for fastmovers

(3) the capacity-availability process for fastmovers.

First we consider the distribution of the aggregate inventory position over the in- dividual products. It proves to be very difficult to determine the conditional probability of the distribution of the individual inventory positions, given the aggregate inventory position. However, since only the aggregate inventory position is to be uscd to determine the production decision, it has to be so that the aggregate inventory position is a good measure for the state of the system. This will not be the case if the inventory position for one fastmover is very negative and for another positive. Therefore we will use $\Sigma_{j=1}^{N_{a}} I_{j}^{+} \cong\left(\Sigma_{j=1}^{N_{a}} I_{j}\right)^{+}$and $\Sigma_{j=1}^{N_{a}} I_{j}^{-} \cong$ $\left(\Sigma_{j=1}^{N_{a}} I_{j}\right)^{-}$to approximate the cost

$$
\begin{aligned}
a & \cdot \Sigma_{j=1}^{N_{a}} I_{j}^{+}+b \cdot \Sigma_{j=1}^{N_{a}} I_{j}^{-} \text {by } a \cdot\left(\Sigma_{j=1}^{N_{a}} I_{j}\right)^{+}+b \cdot\left(\Sigma_{j=1}^{N_{a}} I_{j}\right)^{-} \\
& =a \cdot I^{+}+b \cdot I^{-}
\end{aligned}
$$

(with $I=\Sigma_{j=1}^{N_{a}} I_{j}$ ). This has proven to be a good approximation in the case of identical products (see e.g. Bemelmans and Wijngaard [2]).

The demand process for fastmovers is the superposition of $N_{a}$ independent Poisson processes and, therefore, again is a Poisson process but now with parameter $\lambda_{a}=\Sigma_{j=1}^{N_{a}} \lambda / j$.

The process according to which capacity becomes available for fastmovers is no longer the original Poisson process with parameter $\mu$, since the slowmovers have priority on the capacity and therefore cause a disturbance. Let's first consider the disturbance caused by a single product, say $j$ : As soon as a demand for this slowmover has occurred $q$ times, it will claim the next production instant to start a run (see section 3.1). In this way certain, so-called triggermoments are created for product $j$. It is easy to see that the interarrival time (IAT) between two triggermoments for product $j$ is Erlang-distributed with $q$ phases and parameter $\lambda / j$ (the IAT between two demands for product $j$ has an exponential distribution with parameter $\lambda / j)$.

The process of triggermoments for all 
slowmovers is the superposition of the $N-N_{a}$ individual trigger-processes. Since $N-N_{a}$ (the number of slowmovers) is large, this process will be approximated very well by a Poisson process (see Khintchine [6] for an extensive study on superpositions of non-identical processes). The parameter in this process will be $\frac{\sum_{j=N_{a}+1}^{N} \lambda / i}{q}$.

We are able now to determine the process by-which capacity becomes available for fastmovers. In order to do so we first introduce the stochastic variable $Z(t)$ as the number of slowmovers waiting for production, at time $t$, plus one. So, if no more slowmovers trigger from time $t$ on, then the $Z(t)$ production instant can be used to start a production run for a fastmover. In Fig. 3 we have shown an illustrative example of the behaviour of $Z(t)$.

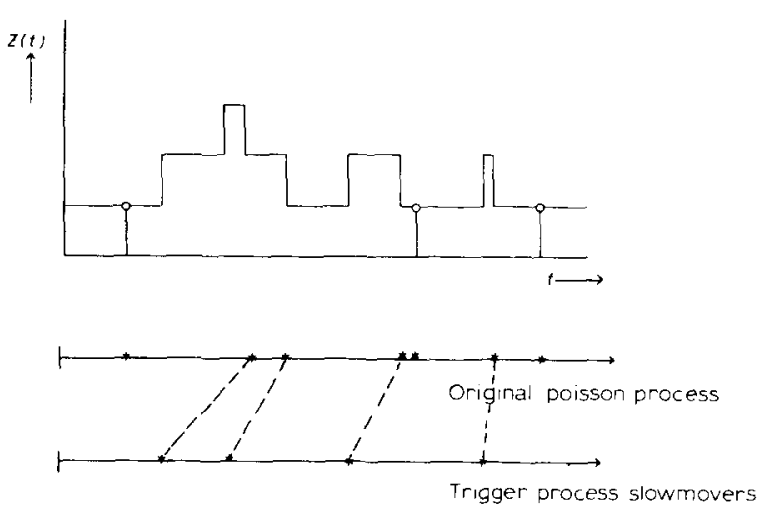

Fig. 3. $Z(t)$ as a function of $t$

A closer consideration of $Z(t)$ makes it clear that $Z(t)$ behaves as the number of customers during a busy period in an $M|M| 1$ queue. This will be clear if one considers the transitions of $Z(t)$ that may occur:

$z \rightarrow z+1:$ if a slowmover triggers (IAT is $\exp \left(\frac{\sum_{j=N_{a}+1}^{N / j}}{q}\right)$ distributed)

$z \rightarrow z-1$ : if a production instant arrives (IAT is $\exp (\mu)$ distributed)

Therefore the IAT between two production instants for fastmovers has the same distribution as the length of a busy period in a $M|M| 1$ queue. This distribution is characterized by e.g. Kleinrock [7].

Let's now return to the determination of $I^{(0)}$. As we have said, this determination depends on three factors. Since we have discussed all these factors, we can now determine $I^{(0)}$. This, for example, can be done by the numerical method that is described by Wijngaard [9]. This method of Wijngaard was developed to calculate total expected cost and related quantities in a countable state Markov chain which is skip-free to the right (that means that, from state $n$, transitions can only take place to the states 0,1 , . . ., n+1). Since in our model transitions from inventory position Ipos can only take place to the inventory positions Ipos-1 and Ipos $+q$, the variable $x=I^{(0)}+q-I$ satisfies the conditions of Wijngaard, one has to approximate the distribution of the IAT between two production instants for a fastmover, by a distribution that has a rational Laplace transform (e.g. Phase-type distribution or Cox-distribution). How such an approximation can be done arbitrarily close is shown by Sauer and Chandy [8] for the case of Cox-distributions.

\subsection{Criterion to distinguish between fastmovers and slowmovers}

So far we have described a capacityoriented strategy, once the decomposition between fastmovers and slowmovers has been given. The only problem left now is how to find a good decomposition. Therefore we will use the following intuitive argument that is similar to the one used in Bemelmans and Wijngaard [2] to determine when to use a product-oriented approach and when to use a capacity-oriented approach. Given a decomposition in two classes, we have found for both classes a production strategy based 
on an approximation of the system. The (theoretical) cost, that is found this way, differs from the real cost because:

- for slowmovers, there will sometimes be interference due to the restricted capacity

- for fastmovers, it will sometimes be so that inventories of different products have opposite sign.

Because of these differences the heuristic is not optimal and the theoretical cost for the heuristic is less than the optimal cost. This holds for each possible decomposition. That means that the decomposition for which the theoretical cost is highest gives a cost that one may expect to be the most realistic. Therefore, we may argue that the decomposition is best in this case too. Thus we have found a simple criterion to decompose the products in a class of fastmovers and a class of slowmovers.

\section{REFERENCES}

1 Agnihothri, S., Karmarkar, U.S. and Kubat, P., 1980. Stochastic allocation rules. Working Paper 8015 , Graduate School of Management, University of Rochester.

2 Bemelmans, R.P.H.G. and Wijngaard, J., 1982. Aggregation versus decomposition in the case of a stochastic single-machine multi-product planning problem. Report BDK/ORS/82/09, Eindhoven University of Technology.

3 Bemelmans, R.P.H.G. and Wijngaard, J., 1982. A capacity oriented approach to production and inventory systems. Proceedings of the Second International Symposium on Inventories, Budapest, 1982 (to appear).

4 Bemelmans, R., 1983. A single-machine multi-product planning problem under periodic review. Report BDK/ ORS/83/02, Eindhoven University of Technology.

5 Cohen, J.W., 1976. On Regenerative Processes in Queueing Theory. Springer-Verlag, Berlin and Heidelberg.

6 Khintchine, A.Y., 1960. Mathematical methods in the theory of queueing. In: M.G. Kendall (ed.), Griffin's Statistical Monographs \& Courses. London.

7 Kleinrock, L., 1976. Queueing Systems, Volume I. Wiley, New York.

8 Sauer, C.H. and Chandy, K.M., 1981. Computer Systems Performance Modelling. Prentice-Hall Inc., Englewood Cliffs, NJ.

9 Wijngaard, J., 1982. On the calculation of the total expected cost in skip-free Markov chains: the matrix case. Operations Research Proceedings 1981. Springer Verlag, Berlin and Heidelberg, pp. 449-453. 